\title{
Quantum percolation in granular metals
}

\author{
M. V. Feigel'man, ${ }^{1,2}$ A. S. Ioselevich, ${ }^{1}$ and M. A. Skvortsov ${ }^{1,2, *}$ \\ ${ }^{1}$ L. D. Landau Institute for Theoretical Physics, Moscow 119334, Russia \\ ${ }^{2}$ Materials Science Division, Argonne National Laboratory, Argonne, Illinois 60439, USA
}

(Dated: April 14, 2004)

\begin{abstract}
Theory of quantum corrections to conductivity of granular metal films is developed for the realistic case of large randomly distributed tunnel conductances. Quantum fluctuations of intergrain voltages (at energies $E$ much below bare charging energy scale $E_{C}$ ) suppress the mean conductance $\bar{g}(E)$ much stronger than its standard deviation $\sigma(E)$. At sufficiently low energies $E_{*}$ any distribution becomes broad, with $\sigma\left(E_{*}\right) \sim \bar{g}\left(E_{*}\right)$, leading to strong local fluctuations of the tunneling density of states. Percolative nature of metal-insulator transition is established by combination of analytic and numerical analysis of the matrix renormalization group equations.
\end{abstract}

PACS numbers: 71.30.+h, 64.60.Ak, 73.23.Hk

Introduction.-Low-temperature electron transport in granular metals was intensively studied during last years [1, 2, 3, 4]. It was shown that in the temperature range $T \geq g \delta$ (where $g \gg 1$ is characteristic value of dimensionless intergrain conductance in units of $e^{2} / 2 \pi \hbar$ and $\delta$ is the intragrain level spacing), quantum corrections to conductivity originate mainly from local fluctuations of voltages between neighboring grains. This effect can be treated within Ambegaokar-Eckern-Schoen model [5] and leads to logarithmic temperature dependence of the effective conductance [1]:

$$
g(T)=g_{0}-\frac{4}{z} \ln \frac{g_{0} E_{C}}{T},
$$

where $E_{C} \gg \delta$ is the charging energy of an individual grain, $g_{0}$ is the bare tunneling conductance of intergrain junctions (identical for all junctions), and $z$ is the coordination number of the lattice [6]. The result (11) is valid as long as the renormalized conductance $g(T)$ is large, i.e. down to temperatures $T_{1}=g_{0} E_{C} e^{-z g_{0} / 4}$. It was argued [2, 3] that transition from metal to insulator behavior (MIT, for brevity) occurs at $T \sim T_{1}$ as long as $T_{1} \geq g \delta$. The same conclusion for the two-dimensional (2D) case was reached [4] via instanton analysis.

Although the above results may well be applied to artificial 2D arrays of well-defined tunnel junctions, tunnel conductances $g_{i j}$ are random in granular metals. In this Letter we investigate the role of $g_{i j}$ randomness for energy (temperature) dependent properties of thin granular films, such as macroscopic conductance $g_{\mathrm{eff}}(T)$ and the local tunneling density of states (LTDoS) $\nu_{i}(E)$. Quantum fluctuations lead to suppression of $g_{i j}$ described by the one-loop renormalization group ( $\mathrm{RG}$ ) equation:

$$
\frac{d g_{i j}}{d t}=-2 g_{i j} R_{i j}
$$

where $t(E)=\ln \left(\bar{g}_{0} E_{C} / E\right)$ is the auxiliary RG "time", $\bar{g}_{0}$ being some mean bare conductance, and $R_{i j}$ is the resistance of the network between the points $i$ and $j$. Physically, renormalization of $g_{i j}$ is due to fluctuations a)

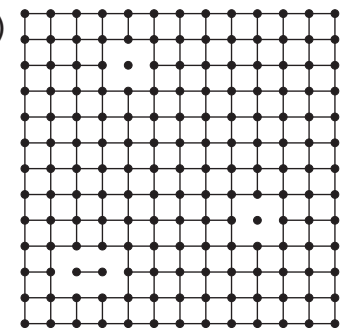

FIG. 1: Conducting bonds (with $g_{i j}>0$ ) at different values of $t$. Results of numerical simulation of Eq. (2) on the lattice $20 \times 20$ with $P_{0}(g)=\left(2 g_{0} \theta(g) / \pi\right) /\left(g^{2}+g_{0}^{2}\right)$. a) $t=0.64 g_{0}$ with the fraction of conducting bonds $N_{\text {cond }}=0.95$; b) $t=1.08 g_{0}$ with $N_{\text {cond }}=0.55$.

of voltage between the grains $i$ and $j$, which are governed by the corresponding resistance $R_{i j}$. Eq. (2) is a straightforward generalization of the $\mathrm{RG}$ equation for a regular array [1] with $g_{i j} \equiv g$ and $R_{i j}=2 / g z$, whose solution is given by Eq. (11). The system of RG equations (2) is nontrivial since $R_{i j}$ is a complicated nonlocal function of all individual conductances $g_{k l}$.

In a regular system, Eq. (2) drives all conductances to zero simultaneously at $t=t_{c}=z g_{0} / 4$, marking the point of the MIT. We will show that in a random system renormalized conductances of the junctions collapse to zero neither all at once, nor one by one, but in groups. These groups enclose clusters, consisting of one or several sites, which become disconnected from the rest of the network after the collapse (see Fig. 1). As a result, the MIT in a natural granular system is a percolative transition: it takes place, when enough clusters have become disconnected so that the percolation via still conducting links is destroyed.

The above picture of conductances, eventually collapsing to zero, follows from the one-loop RG equation (2). The one-loop approximation breaks down at $g \sim 1$; at lower energies the conductance decays exponentially with the RG "time" $t(E)$. Therefore, Eq. (2) can adequately describe only the initial stage of the MIT. Nevertheless, 
there exists a region near the transition where the percolative cluster contains good conductances with $g>1$ so that Eq. (2) is still applicable.

We start from the case of relatively narrow original distribution $P_{0}(g)$ characterized by the mean value $\bar{g}_{0}$ and the standard deviation $\sigma_{0} \ll \bar{g}_{0}$, and show that the renormalized distribution $P(g)$ broadens. In particular, for the square lattice $(z=4)$ :

$$
\frac{\sigma(E)}{\bar{g}(E)}=\frac{\sigma_{0}}{\bar{g}_{0}} \frac{\bar{g}_{0} / \bar{g}}{\sqrt{2 \ln \left(\bar{g}_{0} / \bar{g}\right) \ln \ln \left(\bar{g}_{0} / \bar{g}\right)}},
$$

where $\bar{g} \equiv \bar{g}(E)=\bar{g}_{0}-\ln \left(\bar{g}_{0} E_{C} / E\right)$. Eq. (3) is a large$\ln \left(\bar{g}_{0} / \bar{g}\right)$ asymptotics of a more general expression (see Eq. (11) below). It is valid as long as $\sigma(E) \ll \bar{g}(E)$, i.e. above $E_{*}=T_{*}=\bar{g}_{0} E_{C} e^{-\bar{g}_{0}+\sigma_{0}} \gg T_{1}$, where $T_{1}$ marks MIT in an ideal array with $\sigma_{0} \ll 1$. Thus transition from metal into insulator in a granular array is intrinsically inhomogeneous. The vicinity of this transition at $\max (E, T) \leq T_{*}$ is difficult for exact analytical treatment as the width of distribution $P(g \mid E)$ becomes of order of its mean value. In this region we employ the effectivemedium approximation (EMA) and numerical solution of the RG equations (2), and demonstrate that MIT is of percolative nature.

Strong self-developed inhomogeneity of a granular array can be probed by scanning tunneling measurement of the LTDoS modified by the Coulomb zero-bias anomaly (ZBA) 7, 8, 9]. ZBA modification $Z(E)=\nu(E) / \nu_{0}$ of the average LTDoS in a regular array was considered in Refs. 1, 3] and found to become very large before approaching MIT. Here we analyze spatial fluctuations of the ZBA suppression factor $Z_{i}(E)$. For an originally narrow distribution $P_{0}(g)$, the log-normal distribution of the ZBA factors is found, with $\operatorname{std}\left[\ln Z_{i}(E)\right] \approx \sigma(E) / \bar{g}(E)$. Thus we predict order-of-unity local fluctuations of LTDoS at $\max (E, T) \leq T_{*}$. Spatial correlation length $\xi(E)$ of these fluctuations was found to grow moderately with $E$ decrease in the case of weak original disorder: $\xi(E) \approx \sqrt{\ln \left[\bar{g}_{0} / \bar{g}(E)\right]}$, reaching $\sqrt{\ln \left(\bar{g}_{0} / \sigma_{0}\right)}$ at the border of strong inhomogeneity $E \sim T_{*}$. For the region in the vicinity of MIT, where relative fluctuations are large, we present numerical analysis of LTDoS fluctuations. Below we provide brief derivation of our results.

Narrow distribution.-If the standard deviation $\sigma$ of the distribution is much smaller than the mean $\bar{g}$, the latter follows the homogeneous solution (11): $\bar{g}(t)=\bar{g}_{0}-t$, while evolution of $\delta g_{i j}=g_{i j}-\bar{g}$ can be described perturbatively. Resistance can be written as $R_{i j}=G_{i i}+G_{j j}-$ $2 G_{i j}$, where $G_{i j}=\hat{A}_{i j}^{-1}$ is the Green function of the diffusion operator on the network defined by the matrix elements $A_{i i}=\sum_{j} g_{i j}$ and $A_{i j}=-g_{i j}$ [10]. Using the standard perturbative series $G_{i j}=\bar{G}_{i j}-\bar{G}_{i k} \delta A_{k l} \bar{G}_{l j}+\ldots$ we find

$$
\delta G_{i j}=-\sum_{\langle k l\rangle} \delta g_{k l}\left(\bar{G}_{i k}-\bar{G}_{i l}\right)\left(\bar{G}_{j k}-\bar{G}_{j l}\right),
$$

where in momentum representation $\bar{G}(\mathbf{p})=[2 \bar{g} \varepsilon(\mathbf{p})]^{-1}$; for the square lattice, $\varepsilon(\mathbf{p})=2-\cos p_{x}-\cos p_{y}$.

To proceed further we choose a vector representation for conductances $g_{i}^{\alpha}$ when each edge is characterized by the lattice site $i$ it goes from and direction $\alpha$ which can be either horizontal $(+x)$ or vertical $(+y)$. Using Eq. (4), introducing a new time variable $s=\ln \left[\bar{g}_{0} / \bar{g}(t)\right]=$ $-\ln \left[1-t / \bar{g}_{0}\right]$ and passing to Fourier representation we get a linear evolution:

$$
\frac{d \delta g_{\alpha}(\mathbf{p})}{d s}=-\mathfrak{M}_{\alpha \beta}(\mathbf{p}) \delta g_{\beta}(\mathbf{p}),
$$

governed by the $2 \times 2$ time-independent matrix

$$
\mathfrak{M}(\mathbf{p})=1-\mathcal{P}_{1}(\mathbf{p})-\mathcal{P}_{2}(\mathbf{p})\left(e^{i \alpha_{\mathbf{p}}} \hat{\sigma}_{+}+e^{-i \alpha_{\mathbf{p}}} \hat{\sigma}_{-}\right),
$$

where $\alpha_{\mathbf{p}}=\left(p_{x}-p_{y}\right) / 2$ and $\hat{\sigma}_{ \pm}=\left(\hat{\sigma}_{1} \mp \hat{\sigma}_{2}\right) / 2, \hat{\sigma}_{k}$ being the Pauli matrices. The functions $\mathcal{P}_{1,2}(\mathbf{p})$ are given by

$$
\begin{gathered}
\mathcal{P}_{1}=2 \int(d \mathbf{q}) \frac{\left(1-\cos q_{x}\right)\left(1-\cos \left(p_{x}-q_{x}\right)\right)}{\varepsilon(\mathbf{q}) \varepsilon(\mathbf{p}-\mathbf{q})}, \\
\mathcal{P}_{2}=2 \int(d \mathbf{q}) \frac{\left(\cos \frac{p_{x}}{2}-\cos q_{x}\right)\left(\cos \frac{p_{y}}{2}-\cos q_{y}\right)}{\varepsilon(\mathbf{q}+\mathbf{p} / 2) \varepsilon(\mathbf{q}-\mathbf{p} / 2)},
\end{gathered}
$$

where the integral with $(d \mathbf{q}) \equiv d^{2} q /(2 \pi)^{2}$ runs over the Brillouin zone. At small $\mathbf{p}$ they have a nonanalytic behavior: $\mathcal{P}_{1}(\mathbf{p})=1-1 / \pi-\left(p^{2} / 8 \pi\right) \ln (1 / p)+\ldots$ and $\mathcal{P}_{2}(\mathbf{p})=1 / \pi-\left(p^{2} / 8 \pi\right) \ln (1 / p)+\ldots$

The eigenvalues of the matrix (6) form two branches:

$$
\lambda_{ \pm}(\mathbf{p})=1-\mathcal{P}_{1}(\mathbf{p}) \pm \mathcal{P}_{2}(\mathbf{p})
$$

the eigenfunctions being $\left(e^{i \alpha_{\mathbf{p}}}, \mp 1\right)^{T} / \sqrt{2}$. The spectral branch $\lambda_{+}(\mathbf{p})$ is gapped whereas the branch $\lambda_{-}(\mathbf{p})$ becomes gapless in the long wave-length limit: $\lambda_{-}(\mathbf{p} \rightarrow$ $0) \approx\left(p^{2} / 4 \pi\right) \ln (1 / p)$. Once the spectral properties of the matrix $\mathfrak{M}$ are known one can express $\delta g$ 's at time $s$ via their initial values at $s=0$ :

$$
\delta g_{\alpha}(\mathbf{r}, s)=\sum_{\mathbf{r}^{\prime}} K_{\alpha \beta}\left(\mathbf{r}-\mathbf{r}^{\prime}, s\right) \delta g_{\beta}\left(\mathbf{r}^{\prime}, 0\right) .
$$

The Fourier-transformed kernel is given by

$$
K(\mathbf{p}, s)=K_{1}(\mathbf{p}, s)+K_{2}(\mathbf{p}, s)\left(e^{i \alpha_{\mathbf{p}}} \hat{\sigma}_{+}+e^{-i \alpha_{\mathbf{p}}} \hat{\sigma}_{-}\right)
$$

where $K_{1,2}(\mathbf{p}, s)=\left(e^{-\lambda_{-}(\mathbf{p}) s} \pm e^{-\lambda_{+}(\mathbf{p}) s}\right) / 2$.

Equation (9) allows to find the evolution of the singlesite distribution function $P(g)$. A more convenient quantity is the characteristic function $\chi(\lambda)$ defined as $e^{-\chi(\lambda)}=\int P(\delta g) e^{i \lambda \delta g} d \delta g$. Assuming that at $s=0$ different conductances are uncorrelated we find for $\chi(\lambda)$ : $\chi(\lambda ; s)=\sum_{\mathbf{r}}\left(\chi\left[K_{1}(\mathbf{r}, s) \lambda ; 0\right]+\chi\left[K_{2}(\mathbf{r}, s) \lambda ; 0\right]\right)$. Thus, the variance of the distribution will decay as

$$
\frac{\sigma^{2}(s)}{\sigma_{0}^{2}}=\frac{1}{2} \int(d \mathbf{p})\left[e^{-2 \lambda_{-}(\mathbf{p}) s}+e^{-2 \lambda_{+}(\mathbf{p}) s}\right] .
$$




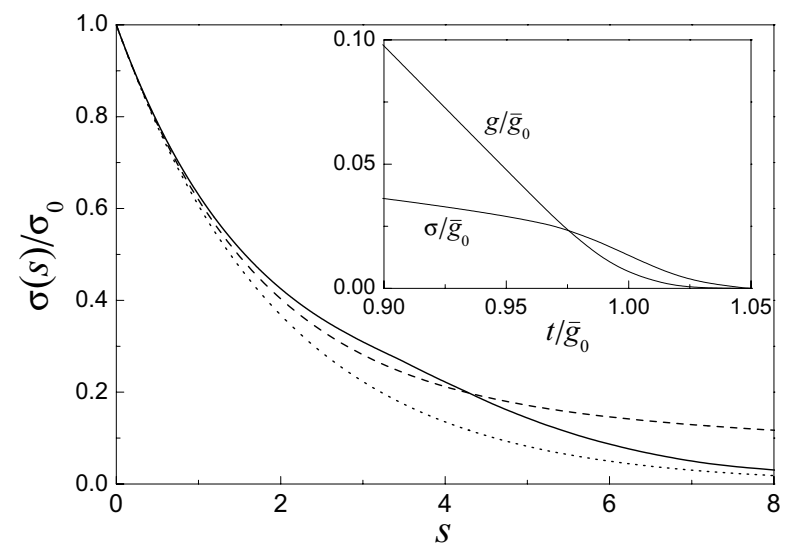

FIG. 2: Evolution of $\sigma(s) / \sigma_{0}$, Eq. (11), (dashed line), the result of numerical simulation of Eq. (2) with $\sigma_{0} / \bar{g}_{0}=0.1$ (solid line), and the EMA prediction $e^{-s / 2}$ (dotted line). Inset: $\bar{g}(t)$ and $\sigma(t)$ near the MIT.

For $s \ll 1, \sigma(s) / \sigma_{0}=1-s / 2+\ldots$ This series is to be compared with decay of the mean conductance: $\bar{g}(s) / \bar{g}_{0}=e^{-s}=1-s+\ldots$ Thus, even at the initial stage of the evolution the width of the distribution decays slower than its average. In the case $s \gg 1$ the integral (11) is dominated by the soft mode $\lambda_{-}(\mathbf{p})$ at $\mathbf{p} \rightarrow 0$ leading to $\sigma^{2}(s) / \sigma_{0}^{2} \approx 1 /(2 s \ln s)$ and hence to Eq. (3). Practically, the applicability of this asymptotics is limited to very large $s$. For intermediate values of $s$ one has to employ full Eq. (11) with numerical integration over the Brillouin zone. The obtained function $\sigma(s) / \sigma_{0}$ together with the prediction of the EMA and results of numerical simulation of Eq. (2) [for a typical realization of disorder on the $20 \times 20$ lattice with periodically boundary conditions and $\left.P_{0}(g)=\left(2 g_{0} \theta(g) / \pi\right) /\left(g^{2}+g_{0}^{2}\right)\right]$ is shown in Fig. [2]

Apart from broadening the single-site distribution $P(g)$, the RG flow (2) produces correlations between $\delta g$ at different links: $C_{\alpha \beta}\left(\mathbf{r} ; s_{1}, s_{2}\right)=\left\langle\delta g_{\alpha}\left(\mathbf{r}, s_{1}\right) \delta g_{\beta}\left(0, s_{2}\right)\right\rangle$. The Fourier transform of the correlation function reads:

$$
C\left(\mathbf{p} ; s_{1}, s_{2}\right)=\sigma_{0}^{2} K\left(\mathbf{p} ; s_{1}+s_{2}\right) .
$$

At the initial stage of evolution, at $s \equiv\left(s_{1}+\right.$ $\left.s_{2}\right) / 2 \lesssim 1$, correlations are short-ranged. At the later stage, $s \gtrsim 1$, correlations with large correlation length $\xi(s)=\sqrt{(4 / \pi) s \ln s}$ develop: $C_{\alpha \beta}\left(\mathbf{r}, s_{1}, s_{2}\right)=$ $\sigma^{2}(s) \exp \left[-r^{2} / \xi^{2}(s)\right]$.

Spatial fluctuations of $g_{i j}$ lead to fluctuations of the LTDoS $\nu_{i}(E)=Z_{i}(E) \nu_{0}$. The ZBA suppression factor $Z_{i}(E)$ for granular media at $E \geq g \delta$ can be found according to simple "environmental theory" [1]]:

$$
\ln Z_{i}(E)=-2 \int_{0}^{t} R_{i}\left(t^{\prime}\right) d t^{\prime}
$$

where $R_{i}(t)$ is the resistance between the site $i$ and the far

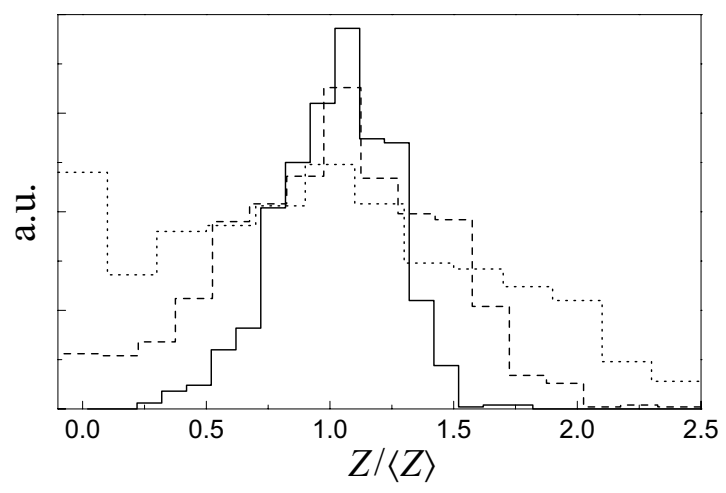

FIG. 3: Histograms for the distribution of the ZBA factors $Z(E)$ near the percolation threshold $t_{c}=0.99 \bar{g}_{0}$ obtained numerically for $\sigma_{0} / \bar{g}_{0}=0.32: t=0.8 t_{c}$ (solid line); $t=0.9 t_{c}$ (dashed line); $t=0.95 t_{c}$ (dotted line).

region of the array at the energy scale $E=\bar{g}_{0} E_{C} e^{-t}$. The same result follows from the analysis provided in 1, 3]. It is important to note that in a homogeneously disordered metal the short-length cutoff in the integral that determines the effective resistance $R(E)$ is given by the diffusion length $\sqrt{\hbar D / E}$, whereas in the present case it is just the grain size. The long-scale cutoff for logarithmic divergency of $R_{i}(E)$ in $2 \mathrm{D}$ is $L(E)=e^{2} g_{\mathrm{eff}} / E$ (in the absence of external screening). Thus one can write $R_{i}(E)=G_{i i}^{\mathrm{reg}}$, where the otherwise divergent $G_{i i}$ is regularized by the finite length $L(E)$. Local fluctuations of $\ln Z_{i}(E)$ are determined by a much smaller region of the size $\xi$ around the site $i$ so that the object $\delta R_{i}=\delta G_{i i}$ is already free of infra-red divergency and is independent on the details of screening. Employing Eq. (4) we obtain

$$
\delta \ln Z_{i}(t)=-2 \int_{0}^{t} d t^{\prime} \sum_{k, \alpha} \frac{\delta g_{\alpha}\left(\mathbf{r}_{k}, t^{\prime}\right)}{g_{0}^{2}\left(t^{\prime}\right)} Q_{\alpha}\left(\mathbf{r}_{i}-\mathbf{r}_{k}\right),
$$

where $Q_{\alpha}(\mathbf{r})$ is specified by its Fourier transform:

$$
Q_{\alpha}(\mathbf{p})=e^{-i p_{\alpha} / 2} \int(d \mathbf{q}) \frac{\cos \left(p_{\alpha} / 2\right)-\cos q_{\alpha}}{2 \varepsilon(\mathbf{q}+\mathbf{p} / 2) \varepsilon(\mathbf{q}-\mathbf{p} / 2)} .
$$

Averaging $\delta \ln Z_{i}(t)$ with the help of Eq. (12), and changing integration variable from $t$ to $s$ we get for the variance of the ZBA exponent:

$$
\begin{aligned}
& \left\langle\left[\delta \ln Z_{i}(t)\right]^{2}\right\rangle=4 \frac{\sigma_{0}^{2}}{\bar{g}_{0}^{2}} \int_{0}^{s} \int_{0}^{s} d s_{1} d s_{2} e^{s_{1}+s_{2}} \\
& \times \int(d \mathbf{p}) K_{\alpha \beta}\left(\mathbf{p} ; s_{1}+s_{2}\right) Q_{\alpha}(\mathbf{p}) Q_{\beta}(-\mathbf{p}) .
\end{aligned}
$$

At the initial stage of the evolution, for $s \lesssim 1$, $\left\langle\left[\delta \ln Z_{i}(t)\right]^{2}\right\rangle \approx\left(0.26 \sigma_{0} / \bar{g}_{0}\right)^{2} s^{2}$. In the region of welldeveloped correlations, for $s \gtrsim 1$, Eq. (16) is dominated by small momenta. In this limit Eq. (15) can be estimated as $Q_{\alpha}(\mathbf{p} \rightarrow 0)=(1 / 4 \pi) \ln (1 / p)$, yielding 
$\left\langle\left[\delta \ln Z_{i}(t)\right]^{2}\right\rangle \approx\left(\sigma_{0}^{2} / 8 \pi^{2} \bar{g}_{0}^{2}\right)\left(e^{2 s} \ln s / s\right)$. Rewriting this result as $\left\langle\left[\delta \ln Z_{i}(t)\right]^{2}\right\rangle \approx(\ln s / 2 \pi)^{2} \sigma^{2}(s) / \bar{g}^{2}(s)$ we see that fluctuations of the ZBA factors $Z_{i}$ become of the order of unity simultaneously with the renormalized ratio $\sigma / \bar{g}$.

The results of numerical simulation for a model distribution $P(g)=\exp \left[-\left(\ln g / \bar{g}_{0}\right)^{2} / 2 \sigma_{1}^{2}\right] /\left(\sqrt{2 \pi} \sigma_{1} g\right)$ with the moderately small variance $\sigma_{0}^{2}=\bar{g}_{0}^{2}\left[e^{2 \sigma_{1}^{2}}-e^{\sigma_{1}^{2}}\right]=$ $\left(0.32 \bar{g}_{0}\right)^{2}$ on the lattice $32 \times 32$ are shown in Fig. 3] where we present the distribution of the local values of $Z_{i}(E)$ at three values of the RG "time" $t$. Upon lowering the energy scale and approaching the MIT transition at $E \sim T_{c}=\bar{g}_{0} E_{C} e^{-t_{c}}$ with $t_{c}=0.99 \bar{g}_{0}$, we observe a growing relative width of $Z$ distribution, with the zero- $Z$ peak developing near the percolation threshold, due to considerable weight of disconnected clusters.

Effective medium approximation (EMA).-In this approximation one takes into account only the simplest local - correlations between $g_{i j}$ and $R_{i j}$, while all distant conductances are replaced with a homogeneous medium with effective conductance $g_{\text {eff }}$ (see, e.g., 12]). Spatial correlation are neglected within EMA, and the system at all "RG times" $t$ is completely described by the singleconductance distribution function $P(g \mid t)$. While being an uncontrolled approximation, EMA provides an instrument to attack the final stage of evolution of any initial distribution - the stage with $\sigma \sim \bar{g}$. We will see that, as it is typical for EMA, it works quite well, except for the immediate vicinity of MIT, for determination of energydependent effective conductance $g_{\text {eff }}(t)$.

Within the EMA,

$$
R_{i j}=\left[g_{i j}+\left(\frac{z}{2}-1\right) g_{\mathrm{eff}}\right]^{-1} .
$$

The effective conductance is then found from the selfconsistency condition 12 ]

$$
\left\langle R_{i j}\left(g_{i j}-g_{\mathrm{eff}}\right)\right\rangle_{g_{i j}}=0 .
$$

Thus, to find $g_{\text {eff }}(t)$ one should, in principle, solve Eqs. (2) and (17) with an arbitrary given $g_{\mathrm{eff}}(t)$ and find $g_{i j}(t)=g\left[g_{i j}(0),\left\{g_{\text {eff }}\right\} \mid t\right]$ as a functional of yet unknown function $g_{\text {eff }}(t)$, and then, finally, solve Eq. (18) for $g_{\text {eff }}(t)$. This leads to a nonlinear integral equation

$$
\int_{0}^{\infty} P_{0}\left(g_{0}\right) d g_{0} \frac{g\left[g_{0},\left\{g_{\mathrm{eff}}\right\} \mid t\right]-g_{\mathrm{eff}}(t)}{g\left[g_{0},\left\{g_{\mathrm{eff}}\right\} \mid t\right]+\left(\frac{z}{2}-1\right) g_{\mathrm{eff}}(t)}=0 .
$$

For a general $P_{0}\left(g_{0}\right)$ this program can be fulfilled only numerically. If the distribution $P_{0}\left(g_{0}\right)$ is narrow, an explicit solution can be obtained for $\delta g_{i j}(t)=g_{i j}(t)-\bar{g}(t)$. For the standard deviation one finds $\sigma_{\mathrm{EMA}}(s) / \sigma(0)=$ $e^{-s(1-2 / z)}$. Comparison of this result (for the square lattice case $z=4$ ) with the exact perturbation theory (11) is shown in Fig. [2 At earlier stages $(s \lesssim 1)$ agreement is rather good, but it becomes worse at large $s$ where pdependence of the eigenvalue $\lambda_{-}(\mathbf{p})$ becomes important.

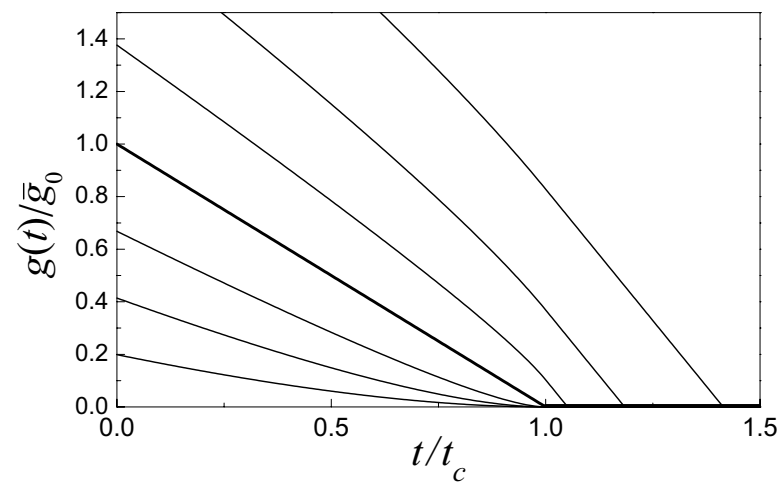

FIG. 4: Evolution of individual conductances according to the effective medium approximation. $g_{\text {eff }}(t)$ is shown with a thick line.

Thus it seems that EMA may work reasonably good for broad distributions when $s$ never becomes large.

An important and physically relevant class of distributions which allow for analytical EMA treatment is defined by the condition that $\ln g$ is symmetrically distributed around some mean value. Writing the tunneling conductance as $g_{i j}=\bar{g}_{0} e^{-h_{i j}}$ one should require the distribution $p_{0}(h)$ of fluctuations $h_{i j}=\kappa\left(d_{i j}-\bar{d}\right)$, where $d_{i j}$ are thicknesses of intergrain insulating barriers, to satisfy $p_{0}(h)=p_{0}(-h)$. For all such distributions on the square lattice $(z=4)$ a simple solution for the effective conductance can be obtained: $g_{\text {eff }}(t)=\bar{g}_{0}-t$ for $t<t_{c}=\bar{g}_{0}$ and $g_{\text {eff }}(t)=0$ at $t \geq t_{c}$. The individual conductances evolve as follows: for $t<t_{c}$,

$$
g_{i j}(t)=\left[\frac{g_{i j}(0)-\bar{g}_{0}}{2 \sqrt{g_{i j}(0)}}+\sqrt{\frac{\left[g_{i j}(0)+\bar{g}_{0}\right]^{2}}{4 g_{i j}(0)}-t}\right]^{2},
$$

while for $t>t_{c}$,

$$
g_{i j}(t)=\left[g_{i j}\left(t_{c}\right)-2\left(t-t_{c}\right)\right] \theta\left[g_{i j}\left(t_{c}\right)-2\left(t-t_{c}\right)\right] .
$$

This evolution is shown in Fig. (4) The straight line $g_{\text {eff }}(t)$ is the separatrix: solutions $g(t)$ with $g(0)>\bar{g}_{0}$ go above $g_{\text {eff }}(t)$ and eventually - one by one - become identical zeros at $t>t_{c}$. Solutions with $g(0)<\bar{g}_{0}$ go below $g_{\text {eff }}(t)$ and become zeros all at once - at $t=t_{c}$, together with $g_{\text {eff }}(t)$. For $t_{c}-t \ll t_{c}$ the latter solutions form a narrow "bunch", manifested by a sharp peak with the total intensity $\approx 1 / 2$ in the distribution function $P(g, t)$ at $g \sim\left(t_{c}-t\right)^{2} / \bar{g}_{0}$. For $t>t_{c}$ this narrow peak transforms into a $\delta$-peak at $g=0$. Its intensity $1-N_{\text {cond }}(t)$ [with $N_{\text {cond }}(t)$ being the fraction of conducting bonds, having $g_{i j}>0$ within the one-loop accuracy of Eq. (2)] jumps from 0 to $1 / 2$ at $t=t_{c}$ and then grows monotonically, approaching unity at $t \gg t_{c}$.

The $t$-dependence of $g_{\text {eff }}(t)$ and $N_{\text {cond }}(t)$ is shown in Fig. [5 together with the results of numerical simulations for the Cauchy initial distribution $P_{0}(g)=\frac{2 \bar{g}_{0} \theta(g)}{\pi\left(g^{2}+\bar{g}_{0}^{2}\right)}$ on 


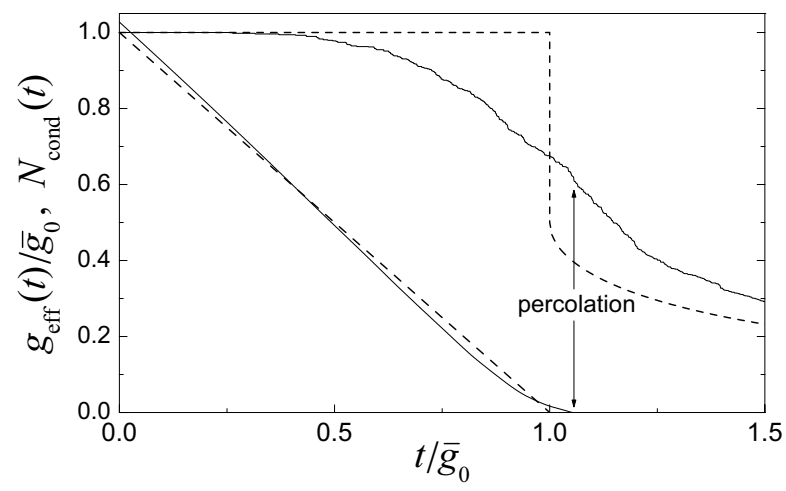

FIG. 5: Comparison of the EMA (dashed lines) and simulation (solid lines) results for the global conductivity $g_{\mathrm{eff}}(t)$ and the fraction of conducting bonds $N_{\text {cond }}(t)$ (see text for details).

the square lattice $32 \times 32$. One can see, that the simulated $g_{\text {eff }}(t)$ follows the EMA in the wide range of $t$, while in the vicinity of the transition it clearly deviates from the EMA and approaches zero with an exponent $\mu>1$. The numerically found $N_{\text {cond }}(t)$ behaves, however, quite smoothly, showing no jump at $t=t_{c}$. The reason for this smoothness is, apparently the tendency to clusterization, demonstrated in Fig. 10 It can be interpreted as an instability, which drives especially small conductances (provided they form an appropriate configuration) to collapse earlier, than typical ones.

Clustering and percolation. - Let us suppose that, due to a fluctuation in the initial distribution and/or to the dynamical evolution up to time $t=t_{0}$, the conductances $g_{i j}\left(t_{0}\right)$ of all bonds within a certain group happen to be much smaller, than conductances in their immediate surrounding. The group should be such that the small conductances belong to the shell of some cluster of sites, separating it from the rest of the array (cf. Fig. (1). Then, for any bond $i j$ from the shell one gets $R_{i j}^{-1} \approx R_{S}^{-1} \equiv \sum_{\text {shell }} g_{k l}$. It means, in particular, that the RG equations for the conductances of the shell are split from the rest of the RG equations, and can be solved separately. As a result, one obtains:

$$
g_{i j}(t) \approx g_{i j}\left(t_{0}\right) \frac{\left(t_{\text {shell }}-t\right) \theta\left(t_{\text {shell }}-t\right)}{t_{\text {shell }}-t_{0}},
$$

where $t_{\text {shell }}-t_{0}=R_{S}^{-1} \ll t_{c}$. Thus, we conclude, that, in contrast to the EMA solution, clusters surrounded by poorly conducting shells may become disconnected from the rest of the array already at $t<t_{c}$.

Numerical simulation clearly demonstrates the formation of clusters (see Fig. 1). When a disconnected cluster appears, the matrix $A$ defined above Eq. (4) acquires a new zero eigenvalue. Thus, the total number of electrically decoupled grains is given by the number of zero eigenvalues of the matrix $A$. The position $t_{c}$ of the percolative transition is a functional of the initial dis- tribution $P_{0}(g)$. Apparently, $t_{c}$ is of the order of some mean initial conductance $\bar{g}_{0}$, while the correct coefficient should be determined numerically.

In general, evolution of the network of conductances with the growth of the parameter $t(E)$ is rather similar to that would be expected at the classical percolation transition. However, our system can not be described by either purely "bond" or "site" percolation, due to development of local correlations (clustering) along with the RG flow. In particular, numerically observed (cf. Fig. (5) value of $N_{\text {cond }}\left(t_{c}\right)$ is clearly larger than $1 / 2$, contrary to expectations for purely bond percolation in 2D. More detailed numerical work is needed to determine the nature of this new kind of percolative transition; in particular, "measurements" of the conductivity exponent $\mu$ (equal to 1.3 in the standard percolation problem [13]) would be very desirable.

Conclusions.-We have shown that at low temperatures strong intrinsic inhomogeneities are developing in granular metal arrays with moderately large random bare conductances $g_{i j} \gg 1$. As a result, the Coulomb-driven metal-insulator transition expected if $g_{0} \leq \ln \left(E_{C} / \delta\right)$ [2, 4] acquires features of percolation transition. Most directly the predicted behavior can be detected by measuring the distribution of the local tunneling density of states at low temperatures. The best object for such a study would be a granular cermet of metal grains in the insulating matrix, like those studied in Refs. 14, 15. In these materials the ratio $E_{C} / \delta$ was about $10^{3}$, indicating the existence of a broad range for logarithmic corrections to conductivity. It is hardly possible that local tunnel conductances in such a granular cermet are all equal; at best, they can be distributed with the width of the order of the mean conductance. Our results presented in Fig. [5 show that a simple logarithmic dependence $g_{\text {eff }}(T)=\bar{g}_{0}-\ln \left(\bar{g}_{0} E_{C} / T\right)$ holds in a wide range of $T$ for moderately random granular arrays as well, at least for the class of practically important symmetric distributions of $\log (g)$ in the 2D space.

If a granular metal has a tendency to become superconductive with $T_{\mathrm{sc}} \sim T_{c}$, its local superconductive properties are expected to be strongly inhomogeneous due to position-dependent Coulomb effects. In other terms, superconductive properties of granular metal can be much more of "granular nature" than its normal properties at elevated temperatures. In this regard we mention very interesting recent experimental results [16].

We are grateful to I. S. Beloborodov, A. M. Finkelstein, A. V. Lopatin, and V. M. Vinokur for useful discussions on initial stage of this project. This research was supported by the Program "Quantum Macrophysics" of the Russian Academy of Sciences, Russian Ministry of Science, RFBR under grants No. 04-02-16348 and 0402-16998, and by the US Department of Energy, Office of Science through contract No. W-31-109-ENG-38. M. A. S. acknowledges financial support from the Dy- 
nasty foundation and the ICFPM.

* Electronic address: skvor@itp.ac.ru

[1] K. B. Efetov and A. Tschersich, Phys. Rev. B 67, 174205 (2003).

[2] I. S. Beloborodov, K. B. Efetov, A. V. Lopatin and V. M. Vinokur, Phys. Rev. Lett. 91, 246801 (2003).

[3] I. S. Beloborodov, A. V. Lopatin, G. Schwiete and V. M. Vinokur, cond-mat/0311512

[4] J. S. Meyer, A. Kamenev and L. I. Glazman, cond-mat/0401343

[5] V. A. Ambegaokar, U. Eckern and G. Schoen, Phys. Rev. Lett. 48, 1745 (1982); G. Schoen and A. D. Zaikin, Phys. Rep. 198, 237 (1990).

[6] Note that in Refs. [1, 2, 3] the dimensional conductance $g$ is normalized to $2 e^{2} / \hbar$, i.e. it differs by the factor $4 \pi$ from the standard definition we use.

[7] B. L. Altshuler and A. G. Aronov, in Electron-electron interactions in disordered solids, edited by A. L. Efros and M. Pollak (North-Holland, Amsterdam, 1985).

[8] A. M. Finkelstein, in Electron liqud in disordered conductors, v. 14 of Soviet Scientific Reviews, edited by I. M. Khalatnikov (Harwood AP, London, 1990).

[9] L. S. Levitov and A. V. Shytov, JETP Letters 66, 214 (1997) and cond-mat/9607136

[10] The matrix $A$ has the zero eigenvalue associated with the shift of all voltages by a constant. Thus, the inverse matrix $G$ does not exist. However, expression for $R_{i j}$ remains well defined since it does not contain the zero mode.

[11] G.-L. Ingold and Yu. V. Nazarov, in Single Electron Tunneling, edited by H. Grabert and M. H. Devoret (Plenum Press, New York and London, 1992).

[12] S. Kirkpatrick, Rev. Mod. Phys. 45, 574 (1973).

[13] P. Grassberger, Physica A 262, 251 (1999); cond-mat/9808095

[14] A. Gerber, A. Milner, G. Deutscher, M. Karpovsky and A. Gladkikh, Phys. Rev. Lett. 78, 4277 (1997).

[15] R. W. Simon, B. J. Dalrimple, D. Van Vechten, W. W. Fuller and S. A. Wolf, Phys. Rev. B 36, 1962 (1987).

[16] W. Escoffier, C. Chapelier, N. Hadacek and J-C. Villegier, cond-mat/0403764 